\title{
Effect of compaction on the degradation of crushed concrete used as partial aggregate substitute in asphalt mixtures
}

\begin{abstract}
The amount of crushed cement concrete continues to grow every day from the demolition of old structures, causing more pollution. Hence, owing to the increase in environmental awareness and stringent regulations governing the disposal, as set by environmental protection agencies, more effective measures for the handling and disposal of crushed concrete must be implemented. Instead of simply disposing of crushed concrete, effective alternative efforts should be considered to utilise it as a reusable material. The objective of this study is to evaluate the possible utilisation of crushed concrete as an aggregate substitute in asphalt mixture for pavement construction applications. However, owing to the poor physical properties of crushed concrete in terms of absorption and abrasion, this study focuses on the degradation that happens in the aggregate after compaction. The recommendation is for crushed concrete to be mixed with conventional aggregates from natural sources. In this study, suitable mixtures of crushed concrete and conventional aggregates were determined based on the combinations of five types of gradation and six different proportions of crushed cement concrete $(0,20,40,60,80$ and 100\%) from an aggregate passing and retained sieve size of $5 \mathrm{~mm}$ and $1.18 \mathrm{~mm}$, respectively. Mixtures of the crushed concrete and conventional aggregates were subjected to compaction of 20, 40, 60, 80 or 100 blows, using a Marshall compactor. Based on the study, the mixtures of the crushed concrete with the recommended ranges of sieve sizes and conventional aggregates are suitable for roads with a medium traffic volume.
\end{abstract}

Keyword: Crushed concrete; Compaction; Asphalt mixtures; Recycling; Degradation; Aggregate gradation; Environment 